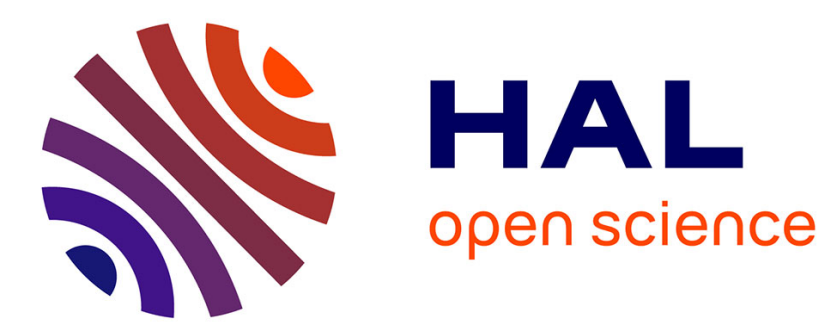

\title{
On the asymptotic distribution of GLR for impropriety of complex signals
}

\author{
Jean-Pierre Delmas, Abdelkader Oukaci, Pascal Chevalier
}

\section{To cite this version:}

Jean-Pierre Delmas, Abdelkader Oukaci, Pascal Chevalier. On the asymptotic distribution of GLR for impropriety of complex signals. Signal Processing, 2011, 91 (10), pp.2259-2267. 10.1016/j.sigpro.2011.04.007 . hal-00664248

\section{HAL Id: hal-00664248 \\ https://hal.science/hal-00664248}

Submitted on 9 Mar 2012

HAL is a multi-disciplinary open access archive for the deposit and dissemination of scientific research documents, whether they are published or not. The documents may come from teaching and research institutions in France or abroad, or from public or private research centers.
L'archive ouverte pluridisciplinaire HAL, est destinée au dépôt et à la diffusion de documents scientifiques de niveau recherche, publiés ou non, émanant des établissements d'enseignement et de recherche français ou étrangers, des laboratoires publics ou privés. 


\title{
On the asymptotic distribution of GLR for impropriety of complex signals
}

\author{
Jean-Pierre Delmas, Abdelkader Oukaci and Pascal Chevalier
}

\begin{abstract}
In this paper, the problem of testing impropriety (i.e., second-order noncircularity) of a sequence of complexvalued random variables (RVs) based on the generalized likelihood ratio test (GLRT) for Gaussian distributions is considered. Asymptotic (w.r.t. the data length) distributions of the GLR are given under the hypothesis that RVs are proper or improper, and under the true, not necessarily Gaussian distribution of the RVs. The considered RVs are independent but not necessarily identically distributed: assumption which has never been considered until now. This enables us to deal with the practical important situations of noncircular RVs disturbed by residual frequency offsets and additive circular noise. The receiver operating characteristic (ROC) of this test is derived as byproduct, an issue previously overlooked. Finally illustrative examples are presented in order to strengthen the obtained theoretical results.
\end{abstract}

\section{Index Terms}

Generalized likelihood ratio (GLR), receiver operating characteristics (ROC), asymptotic distribution of circularity coefficients estimate, improper, second-order noncircular complex random variables.

Revised research paper submitted to Signal Processing.

\section{INTRODUCTION}

For complex-valued RVs, many papers (see, e.g., [1],[2],[3],[4]) show that significant performance gains can be achieved by second-order algorithms based on both $\mathbf{C}_{x}=\mathrm{E}\left(\mathbf{x x}^{T}\right)$ and $\mathbf{R}_{x}=\mathrm{E}\left(\mathbf{x x}^{H}\right)$. They exploit the statistical information contained in $\mathbf{C}_{x}$, provided it is non-zero in addition to that contained in the standard covariance matrix $\mathbf{R}_{x}$. These algorithms face an additional complexity. Moreover, some such algorithms (see e.g., [5]) adapted for improper or second-order noncircular signals, i.e., with non-zero matrices $\mathbf{C}_{x}$, fail or suffer

Jean-Pierre Delmas and Abdelkader Oukaci are with Institut TELECOM, TELECOM SudParis, Département CITI, CNRS UMR 5157, 91011 Evry Cedex, France, e-mail: jean-pierre.delmas(abdelkader.oukaci)@it-sudparis.eu, phone: +(33).1.60.76.46.32, fax: $+(33) .1 .60 .76 .44 .33$.

Pascal Chevalier is with CNAM, CEDRIC laboratory, 75003, Paris France and with Thales-Communications, EDS/SPM, 160 Bd Valmy, 92704 Colombes Cedex, France, e-mail: pascal.chevalier@cnam.fr, phone: +(33).1.40.27.24.85, fax: +(33).1.40.27.24.81. 
of too slow convergence when they are used for proper or second-order circular signals. It is thus important to adapt the processing to the properness of the observation.

Hence, the question arises as to how we can classify a signal as proper or improper. This problem is a binary hypothesis test $H_{0}: \mathbf{C}_{x}=\mathbf{0}$ versus $H_{1}: \mathbf{C}_{x} \neq \mathbf{0}$. In practice, as the parameters $\mathbf{R}_{x}$ and $\mathbf{C}_{x}$ are clearly unknown, only the GLR detector can be used. This detector was introduced independently by Ollila and Koivunen [6] and Schreier, Scharf and Hanssen [7] under the traditional assumption of independent and identically distributed Gaussian samples $\left(\mathbf{x}_{k}\right)_{k=1, \ldots, K}$. But in these works, its performance was illustrated by a Monte Carlo simulation only. Walden and Rubin-Delanchy [8] derived recently this GLRT as well by formulating this testing problem in terms of real-valued Gaussian random vectors. Note that they have also presented a theoretical analysis of the null asymptotic distribution of the GLR with several numerical studies based on Monte Carlo simulations for the alternative distribution under the Gaussian distribution of the signals. Furthermore, there have been recent extensions of this GLRT to non-Gaussian RVs. Authors in [9] have extended this GLRT to complex elliptically symmetric distributions, with a slight adjustment by dividing it with an estimated scaled standardized 4th-order moment. Then in [10], a GLRT based on complex generalized Gaussian distributions have been provided. These extensions make the GLRT more robust to nonGaussian distributions, but surprisingly they do not improve the performance for sub-Gaussian distributions [10], which include the majority of applications in communications and radar.

The aim of this paper is to complement the theoretical asymptotical analysis of [8] and [9]. The originality of our approach consists in considering the null and alternative asymptotic distribution of the GLR derived under the Gaussian distribution, but used in practice under independent not necessarily identically Gaussian distributed data. This paper is organized as follows. The GLRT is recalled for the convenience of the reader. in Section II. The asymptotic distribution of the GLR under the hypothesis that RVs are proper or improper is considered in Section III, using the asymptotic distributions of the circularity coefficients given in [11]. This asymptotic distribution is given in the scalar case and then extended to the multidimensional case under the assumption of independent identically not necessarily Gaussian distributed RVs. An interpretable closed-form expression of the ROC is given in the scalar case due to the simplicity of the asymptotic distribution of the GLR. Then, extension of this study to independent non identically distributed RVs is considered in Section IV. This enables us to deal with practical situations of noncircular RVs disturbed by residual frequency offsets and additive circular noise. Finally some illustrative examples are presented in Section V. Note that some results of this paper have been given in [12].

The following notations are used throughout the paper. Matrices and vectors are represented by bold upper case and bold lower case characters, respectively. Vectors are by default in column orientation, while $T, H$ and $*$ stand for transpose, conjugate transpose, conjugate respectively. $\operatorname{vec}(\cdot)$ is the "vectorization" operator that turns a matrix into a vector by stacking the columns of the matrix one below another which is used in 
conjunction with the Kronecker product $\mathbf{A} \otimes \mathbf{B}$ as the block matrix whose $(i, j)$ block element is $a_{i, j} \mathbf{B}$ and with the vec-permutation matrix $\mathbf{K}$ which transforms $\operatorname{vec}(\mathbf{C})$ to $\operatorname{vec}\left(\mathbf{C}^{T}\right)$ for any matrix $\mathbf{C}$.

\section{Generalized LIKELIHOOD RATIO DECISION RULE}

We assume that $\left(\mathbf{x}_{k}\right)_{k=1, \ldots K} \in \mathbb{C}^{N}$ is a realization of $K$ independent identically zero-mean complex Gaussian distributed RVs. Their covariance matrices $\mathbf{R}_{x}=\mathrm{E}\left(\mathbf{x x}^{H}\right)$ and $\mathbf{C}_{x}=\mathrm{E}\left(\mathbf{x x}^{T}\right)$ are unknown. Consider the following binary composite hypothesis testing problem:

$$
\begin{array}{ll}
H_{0}: & \mathbf{C}_{x}=\mathbf{0}, \quad \mathbf{R}_{x} \\
H_{1}: & \mathbf{C}_{x} \neq \mathbf{0}, \quad \mathbf{R}_{x} .
\end{array}
$$

In the likelihood ratio, the GLR replaces the unknown parameters $\mathbf{R}_{x}$ and $\mathbf{C}_{x}$ by their maximum likelihood (ML) estimates. It is thus straightforward to derive its expression which is given by [6], [7]

$$
L(\mathbf{x}, K) \stackrel{\text { def }}{=} \frac{p\left(\left(\mathbf{x}_{k}\right)_{k=1, \ldots K} ; \widehat{\mathbf{R}}_{x}, \widehat{\mathbf{C}}_{x}, H_{1}\right)}{p\left(\left(\mathbf{x}_{k}\right)_{k=1, \ldots K} ; \widehat{\mathbf{R}}_{x}, \mathbf{0}, H_{0}\right)}=\frac{\operatorname{det}\left(\widehat{\mathbf{R}}_{x}\right)^{K}}{\operatorname{det}\left(\widehat{\mathbf{R}}_{\tilde{x}}\right)^{K / 2}}
$$

with $\widehat{\mathbf{R}}_{x} \stackrel{\text { def }}{=} \frac{1}{K} \sum_{k=1}^{K} \mathbf{x}_{k} \mathbf{x}_{k}^{H}$ and $\widehat{\mathbf{R}}_{\tilde{x}} \stackrel{\text { def }}{=} \frac{1}{K} \sum_{k=1}^{K} \tilde{\mathbf{x}}_{k} \tilde{\mathbf{x}}_{k}^{H}$ where, $\tilde{\mathbf{x}}_{k} \stackrel{\text { def }}{=}\left[\mathbf{x}_{k}^{T}, \mathbf{x}_{k}^{H}\right]^{T}$. The GLRT decides $H_{1}$ if

$$
L(\mathbf{x}, K)>\lambda
$$

and otherwise $H_{0}$. In the scalar case $N=1$, the GLRT is the UMP linearly invariant test [8]. But note that no uniformly most powerful (UMP) $\mathbb{C}$ linearly ${ }^{1}$ invariant test for impropriety exists for $N>1$ [8]. It becomes especially simple

$$
L(\mathbf{x}, K)=\left(1-\widehat{\gamma}_{x}^{2}\right)^{-K / 2}
$$

with $\widehat{\gamma}_{x}=\left|\frac{1}{K} \sum_{k=1}^{K} x_{k}^{2}\right| / \frac{1}{K} \sum_{k=1}^{K}\left|x_{k}\right|^{2}$ is the ML estimate [13], [11] of the circularity coefficient $\gamma_{x} \stackrel{\text { def }}{=}$ $\left|\mathrm{E}\left(x_{k}^{2}\right)\right| / \mathrm{E}\left|x_{k}\right|^{2}$. By the increasing monotony of (3), the GLRT decides $H_{1}$ if

$$
\widehat{\gamma}_{x}>\lambda^{\prime}
$$

which is quite intuitive.

\section{ASYMPTOTIC DISTRIBUTION OF GLR FOR IID OBSERVATIONS}

Throughout this section, this GLRT is used for independent identically zero-mean non necessarily Gaussian distributed RVs $\left(\mathbf{x}_{k}\right)_{k=1, \ldots K}$. For such nonGaussian RVs, decision rule (2) is no longer a GLRT. However, it generally provides good performance in practice (see e.g., for the detection of a known signal corrupted by noncircular interference [14]) and is simple to implement.

\footnotetext{
${ }^{1} \mathbb{C}$ linear transformations include rotation and scaling, but not widely linear operations.
} 


\section{A. Scalar complex random variable}

Let be $x_{k}$ a scalar valued RV of arbitrary distribution with finite 4-th-order moments. We suppose that under $H_{0}, x_{k}$ is circular up to the 4 th-order $^{2}$. Then, the following result is proved in the Appendix:

Result 1: Under the respective hypothesis $H_{0}$ and $H_{1}$, the following convergences in distribution hold when $K \rightarrow \infty$

$$
\begin{gathered}
\sqrt{\frac{K}{1+\frac{\kappa_{x}}{2}}} \widehat{\gamma}_{x} \stackrel{\mathcal{L}}{\rightarrow} \mathcal{R}(1) \\
\sqrt{K}\left(\widehat{\gamma}_{x}-\gamma_{x}\right) \stackrel{\mathcal{L}}{\rightarrow} \mathcal{N}\left(0, \sigma_{\gamma}^{2}\right) \quad \text { if } \quad \gamma_{x} \neq 1 .
\end{gathered}
$$

In (5) and (6), $\mathcal{R}(1)$ and $\mathcal{N}\left(0, \sigma_{\gamma}^{2}\right)$ denote the Rayleigh distribution with unit scale (i.e., the chi distribution with two degrees of freedom $\chi_{2}$ ) and the zero-mean Gaussian distribution with variance $\sigma_{\gamma}^{2}$, respectively, with

$$
\sigma_{\gamma}^{2}=\left(1-\gamma_{x}^{2}\right)^{2}+\gamma_{x}^{2} \kappa_{x}+\frac{\kappa_{x}}{2}+\frac{\gamma_{x}^{2} \Re\left(\kappa_{x}^{\prime}\right)}{2}-2 \gamma_{x}^{2} \Re\left(\kappa_{x}^{\prime \prime}\right) \quad \text { if } \quad \sigma_{\gamma}^{2} \neq 0
$$

where under $H_{0}, \kappa_{x}$ is the normalized-like cumulant $\frac{\operatorname{cum}\left(x_{k}, x_{k}, x_{k}^{*}, x_{k}^{*}\right)}{\left(\mathrm{E}\left(\left|x_{k}\right|^{2}\right)\right)^{2}}$, and under $H_{1}, \kappa_{x}, \kappa_{x}^{\prime}$ and $\kappa_{x}^{\prime \prime}$ are the normalized-like cumulants $\frac{\operatorname{cum}\left(x_{k}, x_{k}, x_{k}^{*}, x_{k}^{*}\right)}{\left(\mathrm{E}\left(\left|x_{k}\right|^{2}\right)\right)^{2}}, \frac{\operatorname{cum}\left(x_{k}, x_{k}, x_{k}, x_{k}\right)}{\left(\mathrm{E}\left(x_{k}^{2}\right)\right)^{2}}$ and $\frac{\operatorname{cum}\left(x_{k}, x_{k}, x_{k}, x_{k}^{*}\right)}{\mathrm{E}\left(\left|x_{k}\right|^{2}\right) \mathrm{E}\left(x_{k}^{2}\right)}$ respectively, which are invariant to any rotation of the distribution of $x_{k}$.

Naturally general expression (7) of $\sigma_{\gamma}^{2}$ simplifies for certain complex distribution classes for which the normalized-like cumulants $\kappa_{x}, \kappa_{x}^{\prime}$ and $\kappa_{x}^{\prime \prime}$ are redundant. For example, the following result is proved in the Appendix.

Result 2: For generalized complex elliptically symmetric distributions (GCES) ${ }^{3}$ introduced in [16] in the multidimensional case, $\sigma_{\gamma}^{2}(7)$ reduces to

$$
\sigma_{\gamma}^{2}=\left(1-\gamma_{x}^{2}\right)^{2}\left(1+\frac{\kappa_{x}}{2+\gamma_{x}^{2}}\right)
$$

Remark 1: This theoretical result means that the estimate $\widehat{\gamma}_{x}$ is approximately Rayleigh (of scale $\frac{1+\frac{\kappa_{x}}{2}}{K}$ ) or Gaussian $\mathcal{N}\left(\gamma_{x}, \frac{\sigma_{\gamma}^{2}}{K}\right)$ distributed under $H_{0}$ and $H_{1}$, respectively for $K \gg 1$. Furthermore the domain of validity of this approximation depends on $\gamma_{x}$ and $\sigma_{\gamma}^{2}$ through the approximate relation $\gamma_{x}-\frac{2 \sigma_{\gamma}}{\sqrt{K}}>0$. For practical use of this result, i.e., for probability of detection $P_{\mathrm{D}} \neq 1$ and probability of false alarm $P_{\mathrm{FA}} \neq 0$, note that the distribution of $\widehat{\gamma}_{x}$ under $H_{0}$ and $H_{1}$ must overlap. This is roughly achieved for $\gamma_{x}-\frac{2 \sigma_{\gamma}}{\sqrt{K}}<\frac{4 \sqrt{1+\kappa_{x} / 2}}{\sqrt{K}}$ as illustrated in Fig.1.

\footnotetext{
${ }^{2}$ This means that not only $\mathrm{E}\left(x_{k}^{2}\right)=0$, but also the fourth-order cumulants satisfy $\operatorname{cum}\left(x_{k}, x_{k}, x_{k}, x_{k}\right)=0$ and cum $\left(x_{k}, x_{k}, x_{k}, x_{k}^{*}\right)=$ 0 [15]. We note, it is possible that $\mathrm{E}\left(x_{k}^{2}\right)=0$ with $\operatorname{cum}\left(x_{k}, x_{k}, x_{k}, x_{k}\right) \neq 0$ or $\operatorname{cum}\left(x_{k}, x_{k}, x_{k}, x_{k}^{*}\right) \neq 0$. In this case, the asymptotic distribution of $\widehat{\gamma}_{x}$ is much more involved (see the proof of Result 1 in the Appendix).

${ }^{3}$ which include the Gaussian distribution.
} 


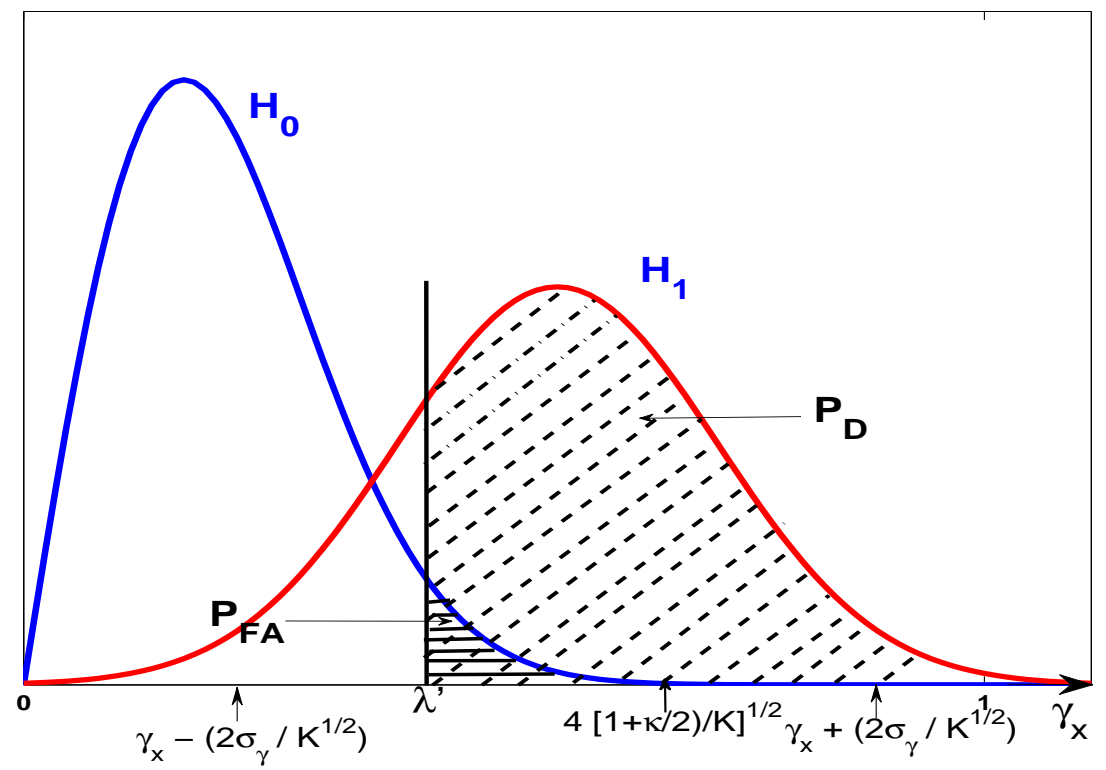

Fig.1 Approximative probability density function (PDF) of $\widehat{\gamma}_{x}$ under $H_{0}$ and $H_{1}$.

Remark 2: For rectilinear RVs, $\gamma_{x}=1$ and thus $x_{k}=r_{k} e^{i \phi}$ where $r_{k}$ is a real-valued RV and with $\phi$ fixed. In this case, the circularity coefficient $\gamma_{x}$ is perfectly estimated, i.e., $\widehat{\gamma}_{x}=1$. Consequently, the detection problem is singular and for a threshold $\lambda^{\prime}$ close to $1, P_{\mathrm{D}}$ and $P_{\mathrm{FA}}$ are equal to 1 and 0 respectively.

Remark 3: Note that $\sigma_{\gamma}^{2}$ can be zero with $\gamma_{x}<1$ (an example of such a situation is given in [11]). In this case, the sequence $K\left(\widehat{\gamma}_{x}-\gamma_{x}\right)$ converges in distribution [17, Th.B, p.124] to a Hermitian form $\mathbf{r}^{H} \boldsymbol{\Omega} \mathbf{r}$, with $\mathbf{r}$ a two dimensional zero-mean complex Gaussian RV,. The distribution of this Hermitian form is defined by the right hand side of (17). But our first order analysis does not allow one to specify the matrix $\Omega$.

Remark 4: Note that for $\gamma_{x}$ close to zero and $K \gg 1,2 \ln L(\mathbf{x}, K)=-K \ln \left(1-\widehat{\gamma}_{x}^{2}\right) \approx K \widehat{\gamma}_{x}^{2}$. Furthermore for Gaussian distributed $x_{k}, \kappa_{x}=0$. In these conditions (5) gives

$$
2 \ln L(\mathbf{x}, K) \stackrel{\mathcal{L}}{\rightarrow} \chi_{2}^{2}, \quad \text { under } H_{0} .
$$

This asymptotic property is consistent with the constant false alarm rate (CFAR) detector where the number 2 of degree of freedom of the chi-squared distribution is equal to the number of real-valued components of $c_{x} \stackrel{\text { def }}{=} \mathrm{E}\left(x_{k}^{2}\right)$, given by the Wilk's theorem $[18, \mathrm{p} .132]^{4}$. But for nonGaussian distributions, detector (4) is no longer asymptotically CFAR. From the practical point of view, similarly as [9], by dividing the test statistic $\widehat{\gamma}_{x}$ with $\sqrt{1+\frac{\widehat{\kappa}_{x}}{2}}$ where $\widehat{\kappa}_{x}$ is any consistent estimate of $\kappa_{x}$, we obtain an adjusted GLRT which becomes asymptotically CFAR. Hence, once the threshold is fixed for a given $P_{\mathrm{FA}}$, the obtained $P_{\mathrm{D}}$ will depend naturally on the unknown parameters $\gamma_{x}$ and $\sigma_{\gamma}^{2}(7)$.

Remark 5: For Gaussian distributed RVs, the normalized-like cumulants $\kappa_{x}, \kappa_{x}^{\prime}$ and $\kappa_{x}^{\prime \prime}$ are zero. Thus the variance $\sigma_{\gamma}^{2}$ of the asymptotic distribution of $\widehat{\gamma}_{x}$ under the hypothesis $H_{1}$ given by (7) and (8) becomes equal

\footnotetext{
${ }^{4}$ Note that this theorem has been used in [8] and [13] for vector and scalar cases to directly derive asymptotic distribution (9).
} 
to $\left(1-\gamma_{x}^{2}\right)^{2}$. It is a decreasing function of $\gamma_{x}$. Consequently for a fixed $P_{\mathrm{FA}}$, i.e., for fixed threshold $\lambda^{\prime}, P_{\mathrm{D}}$ is an increasing function of $\gamma_{x}$ that does not depend on the power of $x_{k}$. This property is very intuitive.

For arbitrary, not necessarily Gaussian distributions of $x_{k}$, Result 1 allows us to derive

$$
\begin{aligned}
P_{\mathrm{FA}} & =P\left(\widehat{\gamma}_{x}>\lambda^{\prime} / H_{0}\right) \approx Q_{\chi_{2}^{2}}\left(\frac{K \lambda^{\prime 2}}{1+\frac{\kappa_{x}}{2}}\right) \\
P_{\mathrm{D}} & =P\left(\widehat{\gamma}_{x}>\lambda^{\prime} / H_{1}\right) \approx Q_{\mathcal{N}}\left(\frac{\sqrt{K}\left(\lambda^{\prime}-\gamma_{x}\right)}{\sigma_{\gamma}}\right),
\end{aligned}
$$

where $Q_{\chi_{2}^{2}}($.$) and Q_{\mathcal{N}}($.$) denote the complementary cumulative distribution functions (i.e., Q_{f}(x) \stackrel{\text { def }}{=}$ $\int_{x}^{+\infty} f(t) d t$ where $f($.$) is the associated probability density function) of the chi-squared distribution with$ 2 degrees of freedom and of the zero-mean, unit-variance Gaussian distribution respectively, and where $\sigma_{\gamma}$ is given by (7). Eliminating the threshold $\lambda^{\prime}$ between $P_{\mathrm{FA}}$ and $P_{\mathrm{D}}$ gives the following closed form expression of the ROC of GLR detector (4)

$$
P_{\mathrm{D}} \approx Q_{\mathcal{N}}\left(\frac{\sqrt{\left(1+\frac{\kappa_{x}}{2}\right) Q_{\chi_{2}^{2}}^{-1}\left(P_{\mathrm{FA}}\right)}-\sqrt{K} \gamma_{x}}{\sigma_{\gamma}}\right) .
$$

From this expression, we clearly see that for fixed $P_{\mathrm{FA}}, P_{\mathrm{D}}$ is an increasing function of the data length $K$ and for Gaussian distributed RVs, an increasing function of the circularity coefficient $\gamma_{x}$.

\section{B. Multidimensional complex random variable}

In the multidimensional case $(N>1)$, Result 1 cannot be easily extended as explained in the Appendix where we can only prove for arbitrary distributions with finite 4-th-order moments the following result.

Result 3: Under hypothesis $H_{1}$, the following convergence in distribution holds when $K \rightarrow \infty$ for the decision statistic $\ell(\mathbf{x}, K) \stackrel{\text { def }}{=}[L(\mathbf{x}, K)]^{-2 / K}$

$$
\sqrt{K}\left(l(\mathbf{x}, K)-\ell_{1}\right) \stackrel{\mathcal{L}}{\rightarrow} \mathcal{N}\left(0, \sigma_{1}^{2}\right) \quad \text { under } \quad H_{1},
$$

where the expressions of $\ell_{1}$ and $\sigma_{1}^{2}$ are derived in the Appendix.

Remark 6: Note that for the Gaussian distribution, i.e., for the only distribution for which the decision statistic $L(\mathbf{x}, K)$ given by (1) is a GLR, Wilk's theorem [18, p.132] applies ${ }^{5}$ and gives

$$
2 \ln L(\mathbf{x}, K) \stackrel{\mathcal{L}}{\rightarrow} \chi_{N(N+1)}^{2} \quad \text { under } H_{0} .
$$

The degree of freedom of the chi-squared distributions is equal to the number $N(N+1)$ of real-valued independent parameters in the Hermitian matrix $\mathbf{C}_{x}$. Under $H_{1}$, in the particular case where $\mathbf{C}_{x}$ is "close" to $\mathbf{0}$ (see a more formal definition in [19, Ch.23.7]), the analysis of [20, Sec.II] is valid and gives the following

\footnotetext{
${ }^{5}$ Note that Wilk's theorem has been invoked in this context in [8] and [9].
} 
approximation of distribution when $K \gg 1$ :

$$
2 \ln L(\mathbf{x}, K) \stackrel{a}{\sim} \chi_{N(N+1)}^{\prime 2}(\mu) \text { under } H_{1}
$$

In this expression, $\chi_{N(N+1)}^{\prime 2}(\mu)$ represents a noncentral chi-squared distribution with $N(N+1)$ degree of freedom and noncentral parameter $\mu$. This parameter is a measure of the discrimination between $H_{0}$ and $H_{1}$. A general expression of this parameter which depends on $K$ is given by [20, exp.(4)].

\section{EXTENSION TO NON IDENTICALLY DISTRIBUTED RVS}

For practical purposes, RVs are not always identically distributed. In particular, when noncircular RVs are disturbed by residual frequency offsets and additive circular noise, RVs could be seen as circular depending on the signal to noise ratio (SNR) and the number $K$ of samples. So in this Section, we still consider the previous GLRT that has been derived under the assumption of independent identically zero-mean complex Gaussian distribution. But it is used here for independent zero-mean non necessarily identically Gaussian distributed RVs ${ }^{6}$ $\left(x_{k}\right)_{k=1, \ldots K}$. To take account of the dependence of the distribution of $x_{k}$ with $k$, the following notation is used: $r_{x, k} \stackrel{\text { def }}{=} \mathrm{E}\left|x_{k}^{2}\right|, c_{x, k} \stackrel{\text { def }}{=} \mathrm{E}\left(x_{k}^{2}\right), \bar{r}_{x, K} \stackrel{\text { def }}{=} \frac{1}{K} \sum_{k=1}^{K} r_{x, k}, \bar{c}_{x, K} \stackrel{\text { def }}{=} \frac{1}{K} \sum_{k=1}^{K} c_{x, k}, \operatorname{cum}_{x, k} \stackrel{\text { def }}{=} \operatorname{cum}\left(x_{k}, x_{k}, x_{k}^{*}, x_{k}^{*}\right)$, $\operatorname{cum}_{x, k}^{\prime} \stackrel{\text { def }}{=} \operatorname{cum}\left(x_{k}, x_{k}, x_{k}, x_{k}\right)$ and $\operatorname{cum}_{x, k}^{\prime \prime} \stackrel{\text { def }}{=} \operatorname{cum}\left(x_{k}, x_{k}, x_{k}, x_{k}^{*}\right)$.

For arbitrary distributions with finite 4th-order moments such that the following Lyapunov conditions [21, Th. 2.7.2] are satisfied $^{7}$

$$
\lim _{K \rightarrow \infty} \frac{\sum_{k=1}^{K} \mathrm{E}|| x_{k}^{2}\left|-r_{x, k}\right|^{3}}{\left(\sqrt{\sum_{k=1}^{K} \mathrm{E}\left(\left(\left|x_{k}^{2}\right|-r_{x, k}\right)^{2}\right)}\right)^{3}}=0 \text { and } \lim _{K \rightarrow \infty} \frac{\sum_{k=1}^{K} \mathrm{E}\left|x_{k}^{2}-c_{x, k}\right|^{3}}{\left(\sqrt{\sum_{k=1}^{K} \mathrm{E}\left(\left(x_{k}^{2}-c_{x, k}\right)^{2}\right.}\right)^{3}}=0,
$$

where $r_{x, k}, c_{x, k}, \operatorname{cum}_{x, k}, \operatorname{cum}_{x, k}^{\prime}$ and $\operatorname{cum}_{x, k}^{\prime \prime}$ are bounded and where we suppose that under $H_{0},\left(x_{k}\right)_{k=1, \ldots, K}$ are circular up to the 4th-order, the following result extending Result 1 is proved in the Appendix.

Result 4: Under the respective hypothesis $H_{0}$ and $H_{1}$, the following convergences in distribution hold when $K \rightarrow \infty$

$$
\begin{gathered}
\sqrt{\frac{K}{\alpha_{K}+\frac{\kappa_{x, K}}{2}}} \widehat{\gamma}_{x} \stackrel{\mathcal{L}}{\rightarrow} \mathcal{R}(1) \\
\sigma_{\gamma, K}^{-1}\left(\widehat{\gamma}_{x}-\gamma_{x, K}\right) \stackrel{\mathcal{L}}{\rightarrow} \mathcal{N}(0,1),
\end{gathered}
$$

where $\alpha_{K} \stackrel{\text { def }}{=} \frac{1}{\bar{r}_{x, K}^{2}} \frac{1}{K} \sum_{k=1}^{K} r_{x, k}^{2}$ and $\kappa_{x, K} \stackrel{\text { def }}{=} \frac{1}{\bar{r}_{x, K}^{2}} \frac{1}{K} \sum_{k=1}^{K} \operatorname{cum}_{x, k}, \gamma_{x, K}$ is the time-averaged circularity coefficient $\frac{\left|\bar{c}_{x, K}\right|}{\bar{r}_{x, K}}=\left|\frac{1}{K} \sum_{k=1}^{K} \mathrm{E}\left(x_{k}^{2}\right)\right| / \frac{1}{K} \sum_{k=1}^{K} \mathrm{E}\left|x_{k}\right|^{2}$ and where the expression of $\sigma_{\gamma, K}$ is derived in the Appendix.

\footnotetext{
${ }^{6}$ We only consider scalar complex-valued RVs, because the extension to multidimensional complex-valued RVs would involve overly too cumbersome notations.

${ }^{7}$ which are not severe and are clearly satisfied for the RVs described by (16).
} 
Remark 7: Clearly for identically distributed RVs, $r_{x, k}=\bar{r}_{x, K}=r_{x}, \frac{\operatorname{cum}_{x, k}}{r_{x}^{2}}=\kappa_{x}$ and thus $\alpha_{K}=1$ and $\kappa_{x, K}=\kappa_{x}$ in (14) and Result 3 reduces to Result 1 under $H_{0}$. Under $H_{1}$, the derivation of $\sigma_{\gamma, K}^{2}(23)$ in the Appendix comes down to the proof of (6), (7) given in [11] for identically distributed RVs where $\sigma_{\gamma, K}=\frac{\sigma_{\gamma}}{\sqrt{K}}$.

\section{ILLUSTRATIVE EXAMPLES}

This section has two purposes. First, we examine the domain of validity of our asymptotic results, and second, we study the performance of the GLR detector in a specific example.

The following MIMO channel (extension of the example given in [7]) that transmits $Q$ independent equiprobable BPSK symbols $a_{q, k} \in\{-1,+1\}$ over an additive noise channel is considered. It also rotates independently the phase of the transmitted symbols $a_{q, k}$ by $\phi_{q, k}$ and are disturbed by residual frequency offsets $\Delta f_{q}$.

$$
\mathbf{x}_{k}=\sum_{q=1}^{Q} \sigma_{q} a_{q, k} e^{i \phi_{q, k}} e^{i 2 \pi k \Delta f_{q}} \mathbf{s}_{q}+\mathbf{n}_{k}
$$

where $\sigma_{q}$ and $\mathbf{s}_{q}$ are $Q$ unknown amplitudes and steering vectors with unit first component. The components of $\mathbf{n}_{k}$ are independent zero-mean complex circular Gaussian RV of unknown variance $\sigma_{n}^{2}$.

We consider three experiments. In the first one, there is no residual frequency offset and under $H_{0}$ and $H_{1}$, we assume that the phase terms $\left(\phi_{q, k}\right)_{k=1, . ., K, q=1, . ., Q}$ are independent and respectively uniformly distributed on $[0,2 \pi]$ or Gaussian distributed with mean $\phi_{q_{0}}$ and variance $\sigma_{\phi_{q}}^{2}$. So we are interested in classifying this channel as either incoherent or partially coherent. This is a binary composite hypothesis testing problem. We easily deduce that

$$
\mathbf{R}_{x}=\sum_{q=1}^{Q} \sigma_{q}^{2} \mathbf{s}_{q} \mathbf{s}_{q}^{H}+\sigma_{n}^{2} \mathbf{I}_{Q} \text { and } \mathbf{C}_{x}= \begin{cases}\mathbf{0} & \text { under } H_{0} \\ \sum_{q=1}^{Q} \sigma_{q}^{2} e^{2 i \phi_{q_{0}}} e^{-2 \sigma_{\phi_{q}}^{2} \mathbf{s}_{q} \mathbf{s}_{q}^{T}} & \text { under } H_{1} .\end{cases}
$$

For $Q=1$ and $N=1, \kappa_{x}=-\frac{1}{\left(1+\rho_{x}^{-1}\right)^{2}}$ under $H_{0}$ and $\gamma_{x}=\frac{e^{-2 \sigma_{\phi_{1}}^{2}}}{1+\rho_{x}^{-1}}, \kappa_{x}=-\frac{1+e^{-4 \sigma_{\phi_{1}}^{2}}}{\left(1+\rho_{x}^{-1}\right)^{2}} \kappa_{x}^{\prime}=e^{-4 \sigma_{\phi_{1}}^{2}}-3$ and $\kappa_{x}^{\prime \prime}=-\frac{2}{1+\rho_{x}^{-1}}$ under $H_{1}$, with an SNR of $\rho_{x} \stackrel{\text { def }}{=} \sigma_{1}^{2} / \sigma_{n}^{2}$.

Fig.2 shows the detection performance $P_{\mathrm{D}}$ for different fixed $P_{\mathrm{FA}}$ for $N=Q=1$ as a function of the SNR for two values of $\sigma_{\phi_{1}}$ deduced from the asymptotic distribution of $\widehat{\gamma}_{x}$ given by Result 1 . We see that the $P_{\mathrm{D}}$ for fixed $P_{\mathrm{FA}}$ is very sensitive to the coherence of the channel. When $\sigma_{\phi_{1}}$ increases for a fixed SNR, the circularity coefficient $\gamma_{x}$ decreases and detection worsens. 


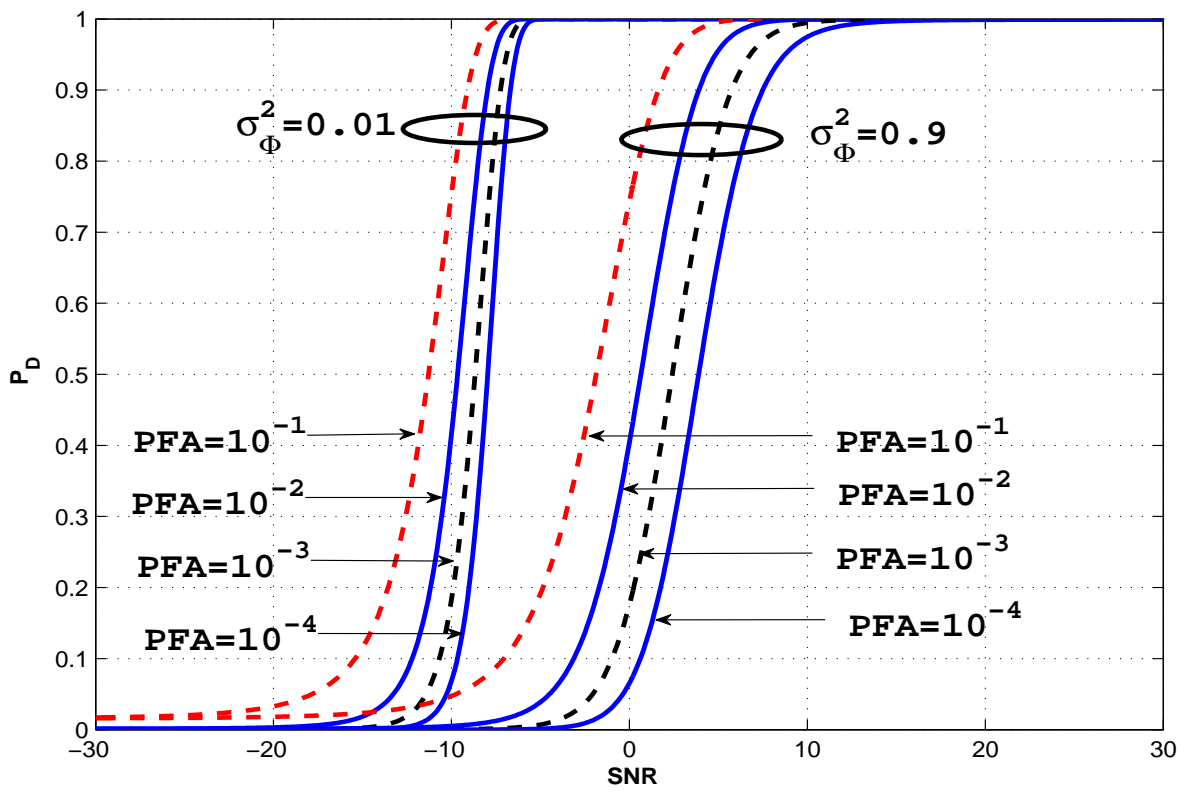

Fig.2 $P_{\mathrm{D}}$ for four different fixed $P_{\mathrm{FA}}$ and two values of $\sigma_{\phi_{1}}$ as a function of SNR for $N=Q=1$ and $K=100$.

In the second experiment, model (16) with $N=Q=1$ is compared to the Gaussian model obtained when $\phi_{1, k}$ does not depend on $k$ and $a_{1, k}$ are independent zero-mean complex circular or real-valued Gaussian RVs under $H_{0}$ and $H_{1}$ respectively. Fig.3 shows the northwest corner of the ROC curve for the GLRT detector for $K=100$ and $\rho_{x}=0.63(-2 d B)$ for BPSK model with a coherent channel (i.e., $\sigma_{\phi_{1}}=0$ ) and Gaussian model, and thus associated with the same value of $\gamma_{x}=0.387$. We note that the ROC curve is sensitive to the distribution of the RVs $x_{k}$, the performance is improved for the BPSK model w.r.t. the Gaussian model and that the empirical ROC fits the asymptotic theoretical ROC for the relatively small data length $K=100$.

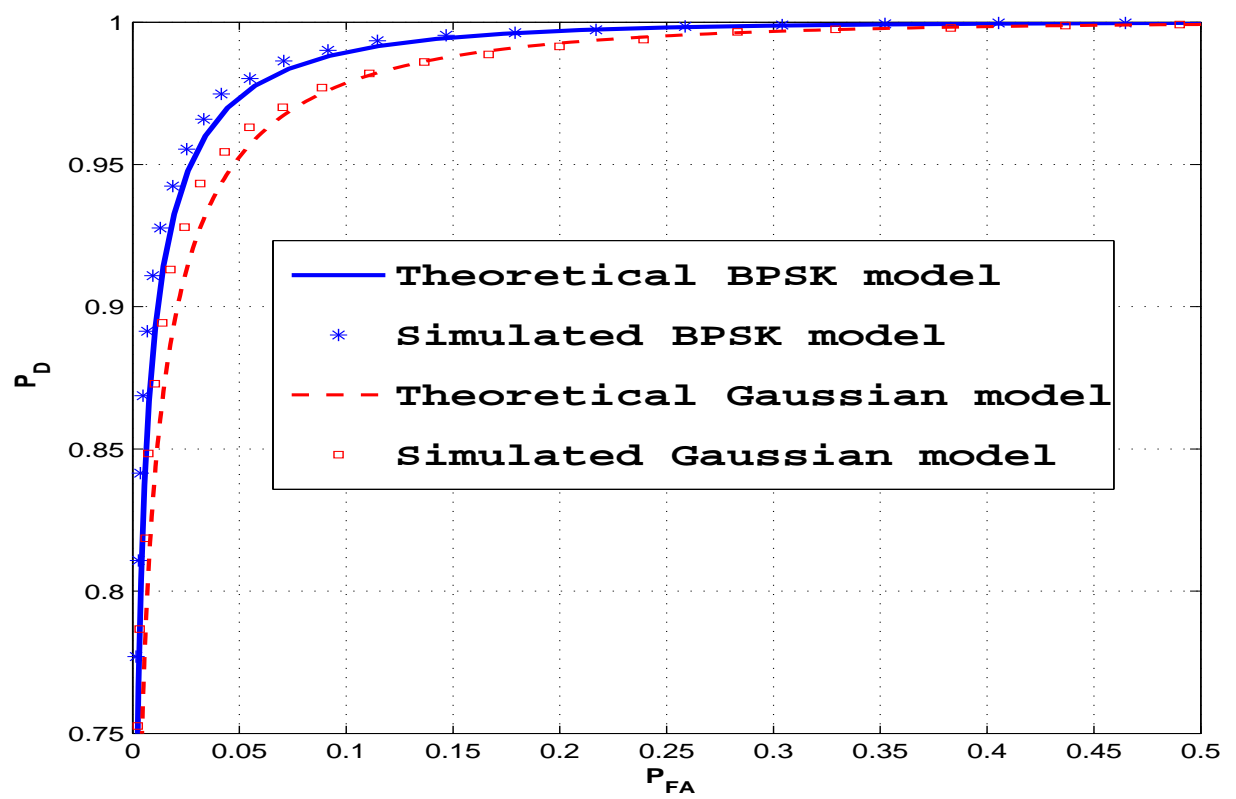

Fig.3 Asymptotic theoretical and empirical (with 10000 Monte Carlo runs) ROC curve associated with BPSK and Gaussian model.

Fig.4 shows the ROC curve for the GLRT detector for the same parameters as in Fig.3, but with 
four residuals of frequency offset $\Delta f_{1}$ for which $r_{x, k}=\bar{r}_{x, K}=\sigma_{1}^{2}+\sigma_{n}^{2}, c_{x, k}=\sigma_{1}^{2} e^{2 i \phi_{1}} e^{4 \pi i k \Delta f_{1}}$, $\bar{c}_{x, K}=\sigma_{1}^{2} e^{2 i \phi_{1}} e^{2 \pi i(K-1) \Delta f_{1}}\left(\frac{\sin 2 \pi K \Delta f_{1}}{\sin 2 \pi \Delta f_{1}}\right)$,

$$
\gamma_{x, K}=\frac{1}{1+\rho_{x}^{-1}} \frac{1}{K}\left|\frac{\sin 2 \pi K \Delta f_{1}}{\sin 2 \pi \Delta f_{1}}\right|
$$

and $\operatorname{cum}_{x, k}=0$ for a Gaussian signal. Comparing to Fig.3, we note in Fig.4 a degradation owing to the frequency offset for which the time-averaged circularity coefficient gets closer to zero under $H_{1}$. The performance of the detector begin decreasing from $K \Delta f_{1}=0.002$ for which $P_{\mathrm{FA}}=0.1$ and 0.05 are obtained for $P_{\mathrm{D}}=0.980$ and 0.960 respectively, against $P_{\mathrm{D}}=0.989$ and 0.970 for no residual frequency offset. The detection capability collapses for $K \Delta f_{1}=0.5$ where the time-averaged circularity coefficient $\gamma_{x, K}=0$. We see also that the empirical ROC fits the asymptotic theoretical ROC for the relatively small data length $K=100$.

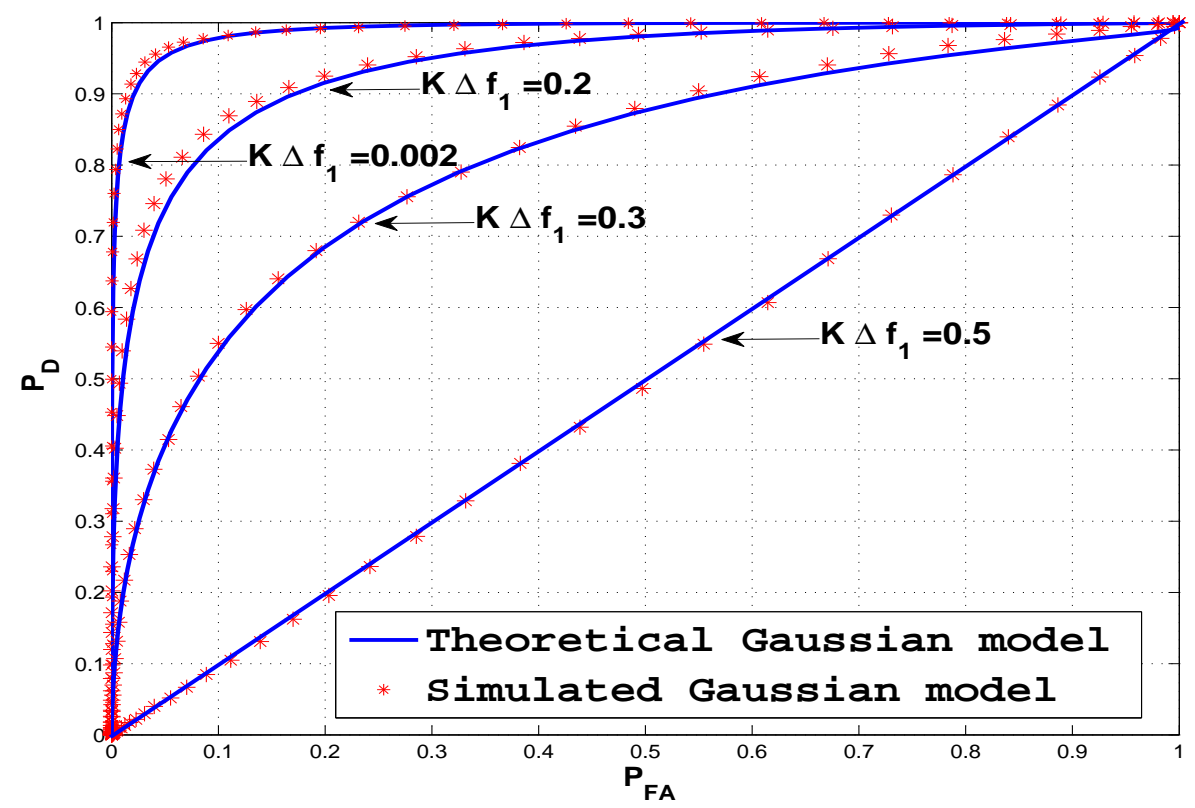

Fig.4 Asymptotic theoretical and empirical (with 10000 Monte Carlo runs) ROC curve associated with Gaussian model for four values of $K \Delta f_{1}$.

Finally in the third experiment, we consider the multidimensional Gaussian $\operatorname{model}^{8}\left(\phi_{q} \stackrel{\text { def }}{=} \phi_{q, k}\right.$ does not depend on $k$ and $a_{q, k}$ are independent zero-mean complex circular or real-valued Gaussian RVs under $H_{0}$ and $H_{1}$ respectively), with no residual of frequency offset. Here, $Q=2, \sigma_{1}=\sigma_{2}$, with an array of $N=2$ omnidirectional sensors equispaced half a wavelength apart. The direction of arrival with respect to broadside of the two sources are $\theta_{1}=0^{\circ}$ and $\theta_{2}=5^{\circ}$. Fig.5 shows the detection performance $P_{\mathrm{D}}$ for different fixed $P_{\mathrm{FA}}$ as a function of the SNR for two values of $\Delta \phi \stackrel{\text { def }}{=} \phi_{1}-\phi_{2} . P_{\mathrm{D}}$ and $P_{\mathrm{FA}}$ are deduced from the asymptotic distribution of $l(\mathbf{x}, K)$ under $H_{1}$ given by Result 2 and of $2 \ln L(\mathbf{x}, K)$ under $H_{0}$ given by (12), respectively. We see that the GLRT is very sensitive to $\Delta \phi$. In particular for very close DOAs (i.e., $\mathbf{s}_{1} \approx \mathbf{s}_{2}$ ) and equipowered

\footnotetext{
${ }^{8}$ We note that in this case under $H_{0}$, the asymptotic distribution of the test statistic is only available for Gaussian distributions of the RVs (see (12). In this case, this test is asymptotically CFAR and once the threshold is fixed for a given $P_{\mathrm{FA}}$, the obtained $P_{\mathrm{D}}$ derived by (11) will depend naturally on the unknown parameters $\ell_{1}=\operatorname{det}\left[\mathbf{I}-\left(\mathbf{R}_{x}^{-1} \mathbf{C}_{x}\right)^{*} \mathbf{R}_{x}^{-1} \mathbf{C}_{x}\right]$ and $\sigma_{1}^{2}$ derived in the Appendix.
} 
sources, $\mathbf{C} \approx \mathbf{0}$ under $H_{1}$ for $\Delta \phi=\pi / 2$ radians, which implies a very bad capability of circularity detection. Furthermore we see that the empirical $\mathrm{P}_{D}$ fits the asymptotic $\mathrm{P}_{D}$ for the relatively small data length $K=100$, except for weak $P_{\mathrm{D}}$.

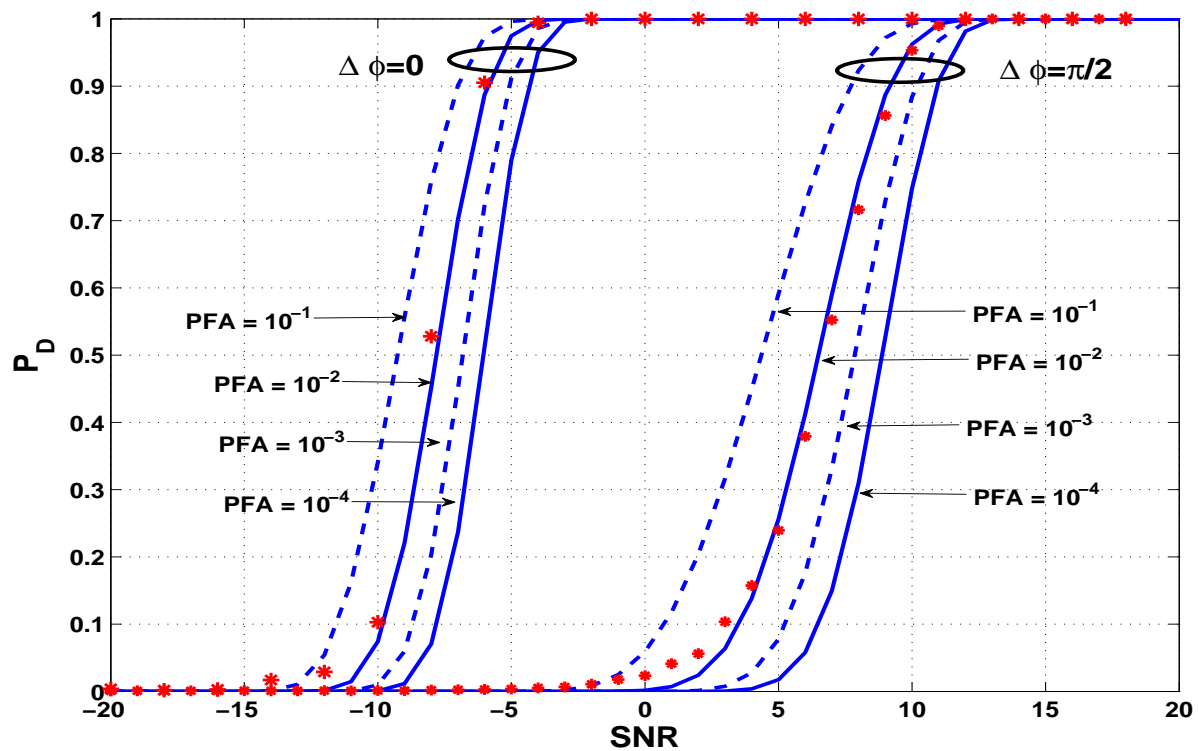

Fig.5 Asymptotic theoretical and empirical (with 10000 Monte Carlo runs) $P_{\mathrm{D}}$ for four different fixed $P_{\mathrm{FA}}$ and two values of $\Delta \phi$ as a function of SNR for $N=Q=2$ and $K=100$.

\section{CONCLUSION}

In this paper, some new enlightening results about the asymptotic distribution of the GLR for impropriety of complex signals have been investigated. The associated GLRT derived under the usual assumption of independent identically distributed Gaussian RVs is studied under non necessarily identical Gaussian distributions of the RVs. For the scalar case, the asymptotic distribution of the circularity coefficient has been given under $H_{0}$ and $H_{1}$ for independent identical or independent non identical arbitrary distributions of the RVs. In particular this allow us to deal with the important practical situations where discrete RVs are disturbed by residual frequency offsets and additive Gaussian circular noise which has never been considered until now. For the multidimensional case, the asymptotic distribution of the GLR has been given under $H_{1}$ for independent and identically arbitrary distributions of the RVs. These results enable us to specify the probability of detection for a specified probability of false alarm, and thus to derive the ROC of this test, an issue previously totally overlooked.

\section{APPENDIX}

Proof of Result 1 Under $H_{1}$, (6) is directly issued form [11, Result 3]. But under $H_{0}$, [11, Result 3] is not valid because it does not holds for $\gamma_{x}=0$. Nevertheless the analysis of [11] still applies. The classical central limit ${ }^{9}$ applied to the independent identically distributed bidimensional complex RVs $\left(\widehat{r}_{x}, \widehat{c}_{x}\right)$ with $\widehat{r}_{x}=\frac{1}{K} \sum_{k=1}^{K}\left|x_{k}^{2}\right|$

\footnotetext{
${ }^{9} \mathcal{N}_{C}(\mathbf{m}, \mathbf{R}, \mathbf{C})$ denotes the complex Gaussian distribution with mean $\mathbf{m}$, and covariances $\mathbf{R}$ and $\mathbf{C}$.
} 
and $\widehat{c}_{x}=\frac{1}{K} \sum_{k=1}^{K} x_{k}^{2}$ yields for $\gamma_{x}=0$

$$
\sqrt{K}\left(\begin{array}{c}
\widehat{r}_{x}-r_{x} \\
\widehat{c}_{x}-c_{x}
\end{array}\right) \stackrel{\mathcal{L}}{\rightarrow} \mathcal{N}_{C}\left(\left(\begin{array}{l}
0 \\
0
\end{array}\right),\left(\begin{array}{cc}
\sigma_{x}^{4}+\operatorname{cum}_{x} & \operatorname{cum}_{x}^{\prime \prime *} \\
\operatorname{cum}_{x}^{\prime \prime} & 2 \sigma_{x}^{4}+\operatorname{cum}_{x}
\end{array}\right),\left(\begin{array}{cc}
\sigma_{x}^{4}+\operatorname{cum}_{x} & \operatorname{cum}_{x}^{\prime \prime} \\
\operatorname{cum}_{x}^{\prime \prime} & \operatorname{cum}_{x}^{\prime}
\end{array}\right)\right),
$$

where $\sigma_{x}^{2} \stackrel{\text { def }}{=} \mathrm{E}\left|x_{k}^{2}\right|, \operatorname{cum}_{x} \stackrel{\text { def }}{=} \operatorname{cum}\left(x_{k}, x_{k}, x_{k}^{*}, x_{k}^{*}\right), \operatorname{cum}_{x}^{\prime} \stackrel{\text { def }}{=} \operatorname{cum}\left(x_{k}, x_{k}, x_{k}, x_{k}\right)$ and $\operatorname{cum}_{x}^{\prime \prime} \stackrel{\text { def }}{=}$ $\operatorname{cum}\left(x_{k}, x_{k}, x_{k}, x_{k}^{*}\right)$. Then, considering the mapping

$$
\left(\widehat{r}_{x}, \widehat{c}_{x}\right) \longmapsto \widehat{m}_{x}=\frac{\widehat{c}_{x}}{\widehat{r}_{x}} \longmapsto \widehat{\gamma}_{x}=\left|\widehat{m}_{x}\right|,
$$

whose differential of the first step is

$$
d m=\frac{1}{r} d c
$$

under $H_{0}$, the standard theorem of continuity (see e.g., [17, Th.A, p.122]) on regular functions of asymptotically Gaussian statistics applies. Consequently, we obtain the following convergence in distribution to a complex zero-mean Gaussian distribution of variance $\frac{1}{\sigma_{x}^{4}}\left(2 \sigma_{x}^{4}+\operatorname{cum}_{x}\right)$ and pseudo variance $\frac{1}{\sigma_{x}^{4}} \operatorname{cum}_{x}^{\prime}$

$$
\sqrt{K}\left(\widehat{m}_{x}-0\right) \stackrel{\mathcal{L}}{\rightarrow} \mathcal{N}_{C}\left(0,2+\frac{\operatorname{cum}_{x}}{\sigma_{x}^{4}}, \frac{\operatorname{cum}_{x}^{\prime}}{\sigma_{x}^{4}}\right) .
$$

This complex Gaussian distribution becomes circular $\left(\operatorname{cum}_{x}^{\prime}=0\right)$ for $x_{k}$ circular up to the 4th-order. With $\widehat{\gamma}_{x}=\left|\widehat{m}_{x}\right|$, convergence in distribution (5) is proved.

\section{Proof of Result 2}

From [16], the GCES distribution of $x_{k}$ is defined in the scalar case from the distribution of the real-valued bivariate RV $\left(\Re\left(x_{k}\right), \Im\left(x_{k}\right)\right)$ which is real elliptical symmetric (RES) distributed. In the zero-mean case, this RES distribution is defined as a linear transform in $\mathbb{R}^{2}$ of a spherically symmetric distribution [22]. Consequently as a linear transform in $\mathbb{R}^{2}$ is equivalent to an $\mathbb{R}$-linear transform in $\mathbb{C}$ [23], $x_{k}$ is zero-mean GCES distributed, if there exist complex valued scalars $a$ and $b$ such that $x_{k}=a u_{k}+b u_{k}^{*}$ where $u_{k}$ is an arbitrary complex circular RV. Consequently the cumulants of $x_{k}$ satisfy the following relations

$$
\begin{aligned}
\operatorname{cum}\left(x_{k}, x_{k}, x_{k}^{*}, x_{k}^{*}\right) & =\left(\left(|a|^{2}+|b|^{2}\right)^{2}+2|a|^{2}|b|^{2}\right) \operatorname{cum}\left(u_{k}, u_{k}, u_{k}^{*}, u_{k}^{*}\right) \\
\operatorname{cum}\left(x_{k}, x_{k}, x_{k}, x_{k}\right) & =6 a^{2} b^{2} \operatorname{cum}\left(u_{k}, u_{k}, u_{k}^{*}, u_{k}^{*}\right) \\
\operatorname{cum}\left(x_{k}, x_{k}, x_{k}, x_{k}^{*}\right) & =3 a b\left(|a|^{2}+|b|^{2}\right) \operatorname{cum}\left(u_{k}, u_{k}, u_{k}^{*}, u_{k}^{*}\right) .
\end{aligned}
$$

Using

$$
\mathrm{E}\left|x_{k}\right|^{2}=\left(|a|^{2}+|b|^{2}\right) \mathrm{E}\left|u_{k}\right|^{2} \text { and } \mathrm{E}\left(x_{k}^{2}\right)=2 a b \mathrm{E}\left|u_{k}\right|^{2}
$$

the normalized-like cumulants $\kappa_{x}^{\prime}$ and $\kappa_{x}^{\prime \prime}$ becomes

$$
\kappa_{x}^{\prime}=\kappa_{x}^{\prime \prime}=\left(\frac{3}{2+\gamma_{x}^{2}}\right) \kappa_{x} .
$$

Plugging these expressions in (7), gives expression (8) of Result 3. 


\section{Proof of Result 3}

With $\ell(\mathbf{x}, K)=\operatorname{det}\left[\mathbf{I}-\left(\widehat{\mathbf{R}}_{x}^{-1} \widehat{\mathbf{C}}_{x}\right)^{*} \widehat{\mathbf{R}}_{x}^{-1} \widehat{\mathbf{C}}_{x}\right]$, from (1) where $\widehat{\mathbf{C}}_{x} \stackrel{\text { def }}{=} \frac{1}{K} \sum_{k=1}^{K} \mathbf{x}_{k} \mathbf{x}_{k}^{T}$, the proof of Result 2 follows the same steps that for Result 1.

Deriving the asymptotic distribution of $\ell(\mathbf{x}, K)$ under $H_{0}$ and $H_{1}$ is based on the following mapping:

$$
\left(\widehat{\mathbf{R}}_{x}, \widehat{\mathbf{C}}_{x}\right) \longmapsto \widehat{\mathbf{M}}_{x}=\widehat{\mathbf{R}}_{x}^{-1} \widehat{\mathbf{C}}_{x} \longmapsto \widehat{\mathbf{\Sigma}}_{x}=\widehat{\mathbf{M}}_{x}^{*} \widehat{\mathbf{M}}_{x} \longmapsto \ell(\mathbf{x}, K)=\operatorname{det}\left[\mathbf{I}-\widehat{\mathbf{\Sigma}}_{x}\right]
$$

Using the asymptotic Gaussian distribution of $\left(\widehat{\mathbf{R}}_{x}, \widehat{\mathbf{C}}_{x}\right)$ [3], [11] derived from the classical central limit theorem, the differential of the different sub mappings of (21), the chain rule and standard properties of the vec operator [24, Ch.2.4], the standard theorem of continuity (see e.g., [17, p. 122]) on regular functions of asymptotically Gaussian statistics applies.

In particular under $H_{0}$, where $\mathbf{x}_{k}$ is circular up to the 4 th-order, the differential of $\mathbf{M}_{x}$ at $\left(\mathbf{R}_{x}, \mathbf{C}_{x}\right)=\left(\mathbf{R}_{x}, \mathbf{0}\right)$ is similarly as (19), given by

$$
\begin{aligned}
d \mathbf{M}_{x} & =-\mathbf{R}_{x}^{-1} d \mathbf{R}_{x} \mathbf{R}_{x}^{-1} \mathbf{C}_{x}+\mathbf{R}_{x}^{-1} d \mathbf{C}_{x}=\mathbf{R}_{x}^{-1} d \mathbf{C}_{x}, \\
\operatorname{vec}\left(d \mathbf{M}_{x}\right) & =\left(\mathbf{I} \otimes \mathbf{R}_{x}^{-1}\right) \operatorname{vec}\left(d \mathbf{C}_{x}\right) .
\end{aligned}
$$

Consequently, (20) becomes here

$$
\sqrt{K}\left(\operatorname{vec}\left(\widehat{\mathbf{M}}_{x}\right)-\mathbf{0}\right) \stackrel{\mathcal{L}}{\rightarrow} \mathcal{N}_{C}\left(\mathbf{0}, \mathbf{R}_{M}, \mathbf{C}_{M}\right)
$$

with

$$
\mathbf{R}_{M}=\left(\mathbf{I} \otimes \mathbf{R}_{x}^{-1}\right) \mathbf{R}_{C}\left(\mathbf{I} \otimes \mathbf{R}_{x}^{-1}\right) \quad \text { and } \quad \mathbf{C}_{M}=\left(\mathbf{I} \otimes \mathbf{R}_{x}^{-1}\right) \mathbf{C}_{C}\left(\mathbf{I} \otimes \mathbf{R}_{x}^{-*}\right)
$$

where $\mathbf{R}_{C}$ and $\mathbf{C}_{C}$ are the covariance matrices of the asymptotic distribution of $\widehat{\mathbf{C}}_{x}$ given [11] by ${ }^{10}$

$$
\mathbf{R}_{C}=\mathbf{R}_{x} \otimes \mathbf{R}_{x}+\mathbf{K}\left(\mathbf{R}_{x} \otimes \mathbf{R}_{x}\right)+\mathbf{Q}_{x} \quad \text { and } \quad \mathbf{C}_{C}=\mathbf{C}_{x} \otimes \mathbf{C}_{x}+\mathbf{K}\left(\mathbf{C}_{x} \otimes \mathbf{C}_{x}\right)+\mathbf{Q}_{x}^{\prime}
$$

for which here $\mathbf{C}_{C}=\mathbf{0}$. Consequently $\mathbf{C}_{M}=\mathbf{0}$ as in the scalar case, $\widehat{\mathbf{M}}_{x}$ is still asymptotically circular Gaussian distributed under $H_{0}$ for $\mathbf{x}_{k}$ circular up to the 4th-order and the differential of the mapping $\widehat{\mathbf{M}}_{x} \longmapsto \widehat{\mathbf{\Sigma}}_{x}$ at $\mathbf{M}_{x}=\mathbf{0}$ is still zero. But in contrast to the scalar case, the derivation of the asymptotic distribution of $\widehat{\boldsymbol{\Sigma}}_{x}$ needs the second differential of this mapping, which is not accessible by our first order analysis.

Under $H_{1}$, with the differential of the mapping $\left(\widehat{\mathbf{R}}_{x}, \widehat{\mathbf{C}}_{x}\right) \longmapsto \widehat{\mathbf{M}}_{x}$ at $\left(\mathbf{R}_{x}, \mathbf{C}_{x}\right)$ derived from (22)

$$
\begin{aligned}
& d \mathbf{M}_{x}=-\mathbf{R}_{x}^{-1} d \mathbf{R}_{x} \mathbf{R}_{x}^{-1} \mathbf{C}_{x}+\mathbf{R}_{x}^{-1} d \mathbf{C}_{x} \\
& \left.\operatorname{vec}(d \mathbf{M})=-\left(\mathbf{C}_{x} \mathbf{R}_{x}^{-T}\right) \otimes \mathbf{R}_{x}^{-1}\right) \operatorname{vec}\left(d \mathbf{R}_{x}\right)+\left(\mathbf{I} \otimes \mathbf{R}_{x}^{-1}\right) \operatorname{vec}\left(d \mathbf{C}_{x}\right) \stackrel{\text { def }}{=} \mathbf{D}_{M, R} \operatorname{vec}\left(d \mathbf{R}_{x}\right)+\mathbf{D}_{M, C} \operatorname{vec}\left(d \mathbf{C}_{x}\right) \\
& { }^{10} \text { where }\left(\mathbf{Q}_{x}\right)_{i+(j-1) K, \kappa+(l-1) K}=\operatorname{cum}\left(x_{k, i}, x_{k, j}, x_{k, \kappa}^{*}, x_{k, l}^{*}\right) \text { and }\left(\mathbf{Q}_{x}^{\prime}\right)_{i+(j-1) K, \kappa+(l-1) K}=\operatorname{cum}\left(x_{k, i}, x_{k, j}, x_{k, \kappa}, x_{k, l}\right) \text { with } \mathbf{x}_{k}= \\
& \left(x_{k, 1}, \ldots, x_{k, N}\right)^{T} .
\end{aligned}
$$


we obtain from the noncircular Gaussian asymptotic distribution of $\left(\widehat{\mathbf{R}}_{x}, \widehat{\mathbf{C}}_{x}\right)$

$$
\sqrt{K}\left(\operatorname{vec}\left(\widehat{\mathbf{R}}_{x}, \widehat{\mathbf{C}}_{x}\right)-\operatorname{vec}\left(\mathbf{R}_{x}, \mathbf{C}_{x}\right)\right) \stackrel{\mathcal{L}}{\rightarrow} \mathcal{N}_{C}\left(\left(\begin{array}{c}
\mathbf{0} \\
\mathbf{0}
\end{array}\right)\left(\begin{array}{cc}
\mathbf{R}_{R} & \mathbf{R}_{R, C} \\
\mathbf{R}_{R, C}^{H} & \mathbf{R}_{C}
\end{array}\right),\left(\begin{array}{cc}
\mathbf{C}_{R} & \mathbf{C}_{R, C} \\
\mathbf{C}_{R, C}^{T} & \mathbf{C}_{C}
\end{array}\right)\right),
$$

whose expressions of $\mathbf{R}_{R}, \mathbf{R}_{R, C}, \mathbf{C}_{R}$ and $\mathbf{C}_{R, C}$ are given in [11], the following convergence in distribution by the standard theorem of continuity (see e.g., [17, Th.A, p. 122])

$$
\sqrt{K}(\operatorname{vec}(\widehat{\mathbf{M}})-\operatorname{vec}(\mathbf{M})) \stackrel{\mathcal{L}}{\rightarrow} \mathcal{N}_{C}\left(\mathbf{0}, \mathbf{R}_{M}, \mathbf{C}_{M}\right),
$$

with

$$
\begin{aligned}
& \mathbf{R}_{M}=\left(\mathbf{D}_{M, R}, \mathbf{D}_{M, C}\right)\left(\begin{array}{cc}
\mathbf{R}_{R} & \mathbf{R}_{R, C} \\
\mathbf{R}_{R, C}^{H} & \mathbf{R}_{C}
\end{array}\right)\left(\begin{array}{c}
\mathbf{D}_{M, R}^{H} \\
\mathbf{D}_{M, C}^{H}
\end{array}\right) \\
& \mathbf{C}_{M}=\left(\mathbf{D}_{M, R}, \mathbf{D}_{M, C}\right)\left(\begin{array}{cc}
\mathbf{C}_{R} & \mathbf{C}_{R, C} \\
\mathbf{C}_{R, C}^{T} & \mathbf{C}_{C}
\end{array}\right)\left(\begin{array}{c}
\mathbf{D}_{M, R}^{T} \\
\mathbf{D}_{M, C}^{T}
\end{array}\right) .
\end{aligned}
$$

Then consider the differential of the mapping $\widehat{\mathbf{M}}_{x} \longmapsto \widehat{\mathbf{\Sigma}}_{x}=\widehat{\mathbf{M}}_{x}^{*} \widehat{\mathbf{M}}_{x}$ at $\mathbf{M}_{x}$

$$
\begin{aligned}
d \boldsymbol{\Sigma}_{x} & =\mathbf{M}_{x}^{*} d \mathbf{M}_{x}+d \mathbf{M}_{x}^{*} \mathbf{M}_{x}, \\
\operatorname{vec}(d \boldsymbol{\Sigma}) & \left.=\left(\mathbf{I} \otimes \mathbf{M}_{x}^{*}\right) \operatorname{vec}\left(d \mathbf{M}_{x}\right)\right)+\left(\mathbf{M}_{x} \otimes \mathbf{I}\right) \operatorname{vec}\left(d \mathbf{M}_{x}^{*}\right) \stackrel{\text { def }}{=} \mathbf{D}_{\Sigma, M} \operatorname{vec}\left(d \mathbf{M}_{x}\right)+\mathbf{D}_{\Sigma, M^{*}} \operatorname{vec}\left(d \mathbf{M}_{x}^{*}\right),
\end{aligned}
$$

which gives the following asymptotic distribution

$$
\sqrt{K}\left(\operatorname{vec}\left(\widehat{\boldsymbol{\Sigma}}_{x}\right)-\operatorname{vec}\left(\boldsymbol{\Sigma}_{x}\right)\right) \stackrel{\mathcal{L}}{\rightarrow} \mathcal{N}_{C}\left(\mathbf{0}, \mathbf{R}_{\Sigma_{x}}, \mathbf{C}_{\Sigma_{x}}\right),
$$

with

$$
\begin{aligned}
& \mathbf{R}_{\Sigma_{x}}=\left(\mathbf{D}_{\Sigma, M}, \mathbf{D}_{\Sigma, M^{*}}\right)\left(\begin{array}{cc}
\mathbf{R}_{M} & \mathbf{C}_{M} \\
\mathbf{C}_{M}^{*} & \mathbf{R}_{M}^{*}
\end{array}\right)\left(\begin{array}{c}
\mathbf{D}_{\Sigma, M}^{H} \\
\mathbf{D}_{\Sigma, M^{*}}^{H}
\end{array}\right) \\
& \mathbf{C}_{\Sigma_{x}}=\left(\mathbf{D}_{\Sigma, M}, \mathbf{D}_{\Sigma, M^{*}}\right)\left(\begin{array}{cc}
\mathbf{C}_{M} & \mathbf{R}_{M} \\
\mathbf{R}_{M}^{T} & \mathbf{C}_{M}^{*}
\end{array}\right)\left(\begin{array}{c}
\mathbf{D}_{\Sigma, M}^{T} \\
\mathbf{D}_{\Sigma, M^{*}}^{T}
\end{array}\right) .
\end{aligned}
$$

Finally, considering the differential of the mapping $\widehat{\boldsymbol{\Sigma}}_{x} \longmapsto \ell(\mathbf{x}, K)=\operatorname{det}\left[\mathbf{I}-\widehat{\boldsymbol{\Sigma}}_{x}\right]$ at $\boldsymbol{\Sigma}_{x}$

$$
d \ell=-\operatorname{det}\left[\mathbf{I}-\boldsymbol{\Sigma}_{x}\right] \operatorname{Tr}\left[\left(\mathbf{I}-\boldsymbol{\Sigma}_{x}\right)^{-1} d \boldsymbol{\Sigma}_{x}\right]=-\operatorname{det}\left[\mathbf{I}-\boldsymbol{\Sigma}_{x}\right] \operatorname{vec}^{T}\left(\left(\mathbf{I}-\boldsymbol{\Sigma}_{x}^{T}\right)^{-1}\right) \operatorname{vec}\left(d \boldsymbol{\Sigma}_{x}\right) \stackrel{\text { def }}{=} \mathbf{D}_{l, \Sigma} \operatorname{vec}\left(d \boldsymbol{\Sigma}_{x}\right)
$$

from [24, Th.1, p.149], the convergence in distribution (11) follows with $\sigma_{1}^{2}=\mathbf{D}_{l, \Sigma} \mathbf{C}_{\Sigma_{x}} \mathbf{D}_{l, \Sigma}^{T}=\mathbf{D}_{l, \Sigma} \mathbf{R}_{\Sigma_{x}} \mathbf{D}_{l, \Sigma}^{H}$ and $\ell_{1}=\operatorname{det}\left[\mathbf{I}-\left(\mathbf{R}_{x}^{-1} \mathbf{C}_{x}\right)^{*} \mathbf{R}_{x}^{-1} \mathbf{C}_{x}\right]<1$ derived from (1).

\section{Proof of Result 4}

To derive the asymptotic distribution of the GLR and then to extend the results of Subsection III-A, we replace the classical central limit theorem with the Lyapunov theorem (see e.g., [21, Th. 2.7.1]) by checking that the Lyapunov conditions (13) are satisfied for the sequence of zero-mean RVs $\left|x_{k}^{2}\right|-r_{x, k}$ and $x_{k}^{2}-c_{x, k}$. In fact the 
Lyapunov theorem ${ }^{11}$ is valid for zero-mean real-valued scalar RVs $u_{k}$. To extend it to the zero-mean complexvalued multidimensional RV $\left(\left|x_{k}^{2}\right|-r_{x, k}, x_{k}^{2}-c_{x, k}\right)$, we must elaborate a little bit. First, the extension of the Lyapunov theorem to zero-mean real-valued multidimensional $\mathrm{RVs} \mathbf{u}_{k}$ is straightforward by application of the Cramer-Wold theorem [21, Th. 5.1.8] for which the sequence $\mathbf{R}_{u, K}^{-1 / 2} \sum_{k=1}^{K} \mathbf{u}_{k}$ converges in distribution to a zeromean, Gaussian distribution $\mathcal{N}_{R}(\mathbf{0}, \mathbf{I})$ where $\mathbf{R}_{u, K}^{1 / 2}$ is an arbitrary square root of $\mathbf{R}_{u, K} \stackrel{\text { def }}{=} \sum_{k=1}^{K} \mathrm{E}\left(\mathbf{u}_{k} \mathbf{u}_{k}^{T}\right)$. Then the Lyapunov theorem applies to the zero-mean complex valued multidimensional RV $\left(\left|x_{k}^{2}\right|-r_{x, k}, x_{k}^{2}-c_{x, k}\right)$, due to isomorphism between $\mathbb{C}$ and $\mathbb{R}^{2}$. Here, using [25, Th. 1], there exists a sequence of $2 \times 2$ matrices $\mathbf{A}_{K}$ such that

$$
\mathbf{A}_{K}^{-1}\left(\begin{array}{c}
\widehat{r}_{x}-\bar{r}_{x, K} \\
\widehat{c}_{x}-\bar{c}_{x, K}
\end{array}\right) \stackrel{\mathcal{L}}{\rightarrow} \mathcal{N}_{C}(\mathbf{0}, \mathbf{I}, \boldsymbol{\Delta})
$$

with $\Delta$ is diagonal such that

$$
\begin{aligned}
\mathbf{A}_{K} \mathbf{A}_{K}^{H} & =\left(\begin{array}{cc}
\mathrm{E}\left|\widehat{r}_{x}-\bar{r}_{x, K}\right|^{2} & \mathrm{E}\left(\widehat{r}_{x}-\bar{r}_{x, K}\right)\left(\widehat{c}_{x}-\bar{c}_{x, K}\right)^{*} \\
\mathrm{E}\left(\widehat{c}_{x}-\bar{c}_{x, K}\right)\left(\widehat{r}_{x}-\bar{r}_{x, K}\right)^{*} & \mathrm{E}\left|\widehat{c}_{x}-\bar{c}_{x, K}\right|^{2}
\end{array}\right) \\
\mathbf{A}_{K} \boldsymbol{\Delta} \mathbf{A}_{K}^{T} & =\left(\begin{array}{cc}
\mathrm{E}\left(\widehat{r}_{x}-\bar{r}_{x, K}\right)^{2} & \mathrm{E}\left(\widehat{r}_{x}-\bar{r}_{x, K}\right)\left(\widehat{c}_{x}-\bar{c}_{x, K}\right) \\
\mathrm{E}\left(\widehat{c}_{x}-\bar{c}_{x, K}\right)\left(\widehat{r}_{x}-\bar{r}_{x, K}\right) & \mathrm{E}\left(\widehat{c}_{x}-\bar{c}_{x, K}\right)^{2}
\end{array}\right),
\end{aligned}
$$

where the terms of those two matrices are given by

$$
\begin{aligned}
\mathrm{E}\left(\widehat{r}_{x}-\bar{r}_{x, K}\right)^{2} & =\frac{1}{K^{2}} \sum_{k=1}^{K}\left(\operatorname{cum}_{x, k}+\left|c_{k}\right|^{2}+r_{k}^{2}\right), \\
\mathrm{E}\left|\widehat{c}_{x}-\bar{c}_{x, K}\right|^{2} & =\frac{1}{K^{2}} \sum_{k=1}^{K}\left(\operatorname{cum}_{x, k}+2 r_{k}^{2}\right), \quad \mathrm{E}\left(\widehat{c}_{x}-\bar{c}_{x, K}\right)^{2}=\frac{1}{K^{2}} \sum_{k=1}^{K}\left(\operatorname{cum}_{x, k}^{\prime}+2 c_{k}^{2}\right), \\
\mathrm{E}\left(\widehat{c}_{x}-\bar{c}_{x, K}\right)\left(\widehat{r}_{x}-\bar{r}_{x, K}\right) & =\frac{1}{K^{2}} \sum_{k=1}^{K}\left(\operatorname{cum}_{x, k}^{\prime \prime}+2 c_{k} r_{k}\right) .
\end{aligned}
$$

Under $H_{0}$ where the moments of $\left(x_{k}\right)_{k=1, \ldots, K}$ are circular up to the 4th-order, $c_{k}=0, \operatorname{cum}_{x, k}^{\prime}=0$ and $\operatorname{cum}_{x, k}^{\prime \prime}=0$, the delta method [21, Ch. 2] derived from the standard theorem of continuity applied to the mapping (18) with the associated differential $d m=-\frac{c}{r^{2}} d r+\frac{1}{r} d c$ gives here $d m=\frac{1}{r} d c$ and after straightforward algebraic manipulations

$$
\sqrt{\frac{1}{\frac{1}{\left(\bar{r}_{x, K}\right)^{2}} \frac{1}{K} \sum_{k=1}^{K}\left(r_{x, k}^{2}+\frac{1}{2} \operatorname{cum}_{x, k}\right)}}\left(\widehat{m}_{x}-\frac{\bar{c}_{x, K}}{\bar{r}_{x, K}}\right) \stackrel{\mathcal{L}}{\rightarrow} \mathcal{N}_{C}(0,1,0) .
$$

With $\gamma_{x, K} \stackrel{\text { def }}{=} \frac{\left|\bar{c}_{x, K}\right|}{\bar{r}_{x, K}}$, which is the time-averaged circularity coefficient, (14) is proved.

In the same way, under $H_{1},(15)$ is derived from the steps of the Appendix of [11] from the delta method

\footnotetext{
${ }^{11}$ that we restate for the ease of the reader. If $u_{k}$ is a sequence of zero-mean scalar real-valued RVs that satisfies

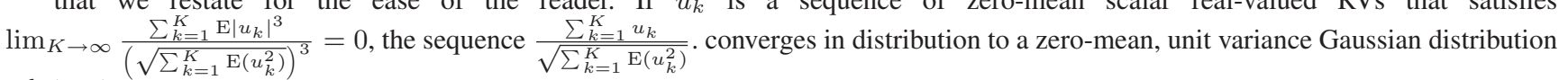
$\mathcal{N}_{R}(0,1)$.
} 
using the two associated differentials

$$
\begin{aligned}
d m & =-\frac{c}{r^{2}} d r+\frac{1}{r} d c \quad \text { and } \quad d \gamma=\frac{1}{2 \gamma}\left(m^{*} d m+m d m^{*}\right), \\
\mathrm{E}\left|\widehat{m}_{x}-\frac{\bar{c}_{x, K}}{\bar{r}_{x, K}}\right|^{2} & =\left(\begin{array}{cc}
-\frac{\bar{c}_{x, K}}{\bar{r}_{x, K}^{2}} & \frac{1}{\bar{r}_{x, K}}
\end{array}\right) \mathbf{A}_{K} \mathbf{A}_{K}^{H}\left(\begin{array}{c}
-\frac{\bar{c}_{x_{2}, K}^{*}}{\bar{r}_{x, K}^{2}} \\
\frac{1}{\bar{r}_{x, K}}
\end{array}\right)+o\left(\frac{1}{K}\right) \stackrel{\text { def }}{=} r_{m, K}+o\left(\frac{1}{K}\right), \\
\mathrm{E}\left(\widehat{m}_{x}-\frac{\bar{c}_{x, K}}{\bar{r}_{x, K}}\right)^{2} & =\left(\begin{array}{ll}
-\frac{\bar{c}_{x, K}}{\bar{r}_{x, K}^{2}} & \frac{1}{\bar{r}_{x, K}}
\end{array}\right) \mathbf{A}_{K} \boldsymbol{\Delta} \mathbf{A}_{K}^{T}\left(\begin{array}{c}
-\frac{\bar{c}_{x, K}}{\bar{r}_{x, K}^{2}} \\
\frac{1}{\bar{r}_{x, K}}
\end{array}\right)+o\left(\frac{1}{K}\right) \stackrel{\text { def }}{=} c_{m, K}+o\left(\frac{1}{K}\right) .
\end{aligned}
$$

Then (15) follows with $\sigma_{\gamma, K}$ is given by

$$
\sigma_{\gamma, K}^{2}=\frac{1}{4 \gamma_{x, K}^{2}}\left(\begin{array}{cc}
\bar{c}_{x, K}^{*} & \bar{c}_{x, K} \\
\overline{r_{x, K}^{*}} & \bar{r}_{x, K}
\end{array}\right)\left(\begin{array}{cc}
r_{m, K} & c_{m, K} \\
c_{m, K}^{*} & r_{m, K}^{*}
\end{array}\right)\left(\begin{array}{c}
\bar{c}_{x, K} \\
\bar{r}_{x, K} \\
\bar{c}_{x, K}^{*} \\
\overline{\bar{r}_{x, K}^{*}}
\end{array}\right) .
$$

\section{REFERENCES}

[1] B. Picinbono and P. Chevalier "Widely Linear Estimation with Complex Data," IEEE Trans. Signal Process., vol. 43, no. 8, pp. 2030-2033, August 1995.

[2] P.J. Schreier, L. Scharf, and C.T. Mullis, "Detection and estimation of improper complex random signals," IEEE Trans. Inform. Theory, vol. 51, no. 1, pp. 306-312, January 2005.

[3] J.P. Delmas, "Asymptotically minimum variance second-order estimation for non-circular signals with application to DOA estimation," IEEE Trans. Signal Process., vol. 52, no. 5, pp. 1235-1241, May 2004.

[4] P. Chevalier and F. Pipon, "New Insights into optimal widely linear array receivers for the demodulation of BPSK, MSK and GMSK signals corrupted by noncircular interferences - Application to SAIC", IEEE Trans. on Signal Processing, vol.54, no.3, pp. 870-883, March 2006.

[5] H. Abeida and J.P. Delmas, "MUSIC-like estimation of direction of arrival for non-circular sources," IEEE Trans. Signal Process., vol. 54, no. 7, pp. 2678-2690, July 2006.

[6] E. Ollila and V. Koivunen, "Generalized complex elliptical distributions," in Proc. 3rd Sensor Array Mutichannel Signal Processing Workshop, Sitges, Spain, July 2004.

[7] P.J. Schreier, L. Scharf, and A. Hanssen, "A generalized likelihood ratio test for impropriety of complex signals," IEEE Signal Process. Letters, vol. 13, no. 7, pp. 433-436, July 2006.

[8] A.T. Walden and P. Rubin-Delanchy, "On testing for impropriety of complex-valued Gaussian vectors," IEEE Trans. Signal Process., vol. 57, no. 3, pp. 825-834, March 2009.

[9] E. Ollila and V. Koivunen, "Adjusting the generalized likelihood ratio test of circularity robust to non-normality," IEEE Int. Workshop on Signal Processing Advances in Wireless Communications (SPAWC 2009), Perugia, Italy, June 2009.

[10] M. Novey, T. Adali, and A. Roy, "Circularity and Gaussianity detection using the complex generalized Gaussian distribution," IEEE Signal Processing Lett., vol. 16, no. 11, pp. 993-996, 2009.

[11] J.P. Delmas and H. Abeida, "Asymptotic distribution of circularity coefficients estimate of complex random variables," Signal Processing (Elsevier), vol. 89, pp. 2670-2675, December 2009.

[12] J.P. Delmas, A. Oukaci, and P. Chevalier, "Asymptotic distribution of GLR for impropriety of complex signals," in Proc. IEEE Int. Conf. Acoustics, Speech and Signal Processing (ICASSP), Dallas, March 2010.

[13] E. Ollila, "On the circularity of a complex random variable," IEEE Signal Process. Letters, vol. 15, pp. 841-844, November 2008.

[14] P. Chevalier, A. Blin, F. Pipon, and F. Delaveau, "GLRT-based array receivers to detect a known signal corrupted by noncircular interferences," in Proc. EUSIPCO, Poznan, Poland, Sept. 2007.

[15] P.J. Schreier and L. Scharf, "Higher-order spectral analysis of complex signals", Signal Processing, no. 86, pp. 3321-3333, 2006.

[16] E. Ollila and V. Koivunen, "Generalized complex elliptical distributions," in Proc. SAM, Sitges, Spain, July 2004.

[17] R.J. Serfling, Approximation Theorems of Mathematical Statistics, John Wiley and Sons, 1980.

[18] G.A. Young and R.L. Smith, Essentials of Statistical Inference, Cambridge Series in Statistical and Probabilistic Mathematics, 2005.

[19] A. Stuart and J.K. Ord, Advanced Theory of Statistics, fifth edition, vol.2, Edward Arnold, 1991.

[20] S.M. Kay, "Asymptotically optimal detection in incompletely characterized non-Gaussian noise," IEEE Trans. ASSP, vol. 37, no. 5, pp. 627-633, May 1989.

[21] E.L. Lehmann, Elements of large sample theory, Springer texts in Statistics, 1998.

[22] K.T. Fang, S. Kotz, and K.W. Ng, Symmetric multivariate and related distributions, Chapman an Hall, London, 1990. 
[23] J. Eriksson, E. Ollila, and V. Koivunen, "Essential statistics and tools for complex random variables," IEEE Transactions on signal processing, vol. 58, no. 10, pp. 5400-5408, October 2010.

[24] J.R. Magnus and H. Neudecker, Matrix differential calculus with applications in statistics and econometrics, Wiley series in probability and statistics, revised edition, 1999.

[25] J. Eriksson and V. Koivunen, "Complex random vectors and ICA Models: Identifiability, uniqueness, and separability," IEEE Trans. on Inform. Theory, vol. 52, no. 3, pp. 1017-1029, March 2006. 